\title{
AUTOMATIC GUIDANCE OF AGRICULTURAL VEHICLES BASED ON GLOBAL POSITIONING SYSTEM
}

\author{
Lan Yao, Li Li, Miao Zhang, Li Minzan \\ Key laboratory of Modern Precision Agriculture System Integration Research, Ministry of \\ Education, China Agriculture University, Beijing 100083, China.Email: limz@cau.edu.cn
}

\begin{abstract}
The need for automatic guidance of farm vehicles has been recognized for a considerable time. Automatic guidance of agricultural vehicles must have some effective method for determining their position in the working environment in order to operate appropriately. Recently, Global Positioning System (GPS) receivers have become available for world-wide and all-weather outdoor localization. This paper presents a review of automatic guidance of agricultural vehicles based on stand-off Global Positioning System (GPS) or the integration of GPS and dead-reckoning system.
\end{abstract}

Key words: Automatic guidance, Global Positioning System, Dead-reckoning system

\section{INTRODUCTION}

Automatic vehicle guidance was defined as 'accurate automatic control of the vehicle or implement along a predefined trajectory ${ }^{11}$. Over the past century, many changes have occurred in agricultural field production machinery ${ }^{[2]}$. The need for automatic guidance of farm vehicles has been recognized for a considerable time.

Automated guidance systems attempt to relieve the operator from many, if not all, of the tasks involved in guiding a vehicle.

There are several benefits of automatic guidance systems in agriculture. One major benefit of automatic guidance systems is a reduction in machine operator fatigue by automating repetitively monotonous steering tasks. And 
it will also complement and augment the farmer's abilities and skills to increase the volume and quality of produce, while production costs (particularly labor costs) are decreased and the working conditions improved.

Another benefit of automatic guidance systems is a potential improvement in the placement of agricultural inputs. With larger equipment, human operators often overlap previous paths in field operations, subjecting parts of a field to a double application of farming inputs (Nieminen and Sampo, 1993) ${ }^{[2]}$.

Furthermore, the potential for vehicle automation in agriculture has increased by the advent of precision agriculture. Precision agriculture has helped advance vehicle guidance firstly in terms of providing position information that is required for vehicle guidance. Secondly, precision agriculture has placed the notion of vehicle automation within the conceptual boundary of equipment manufacturers and agricultural producers ${ }^{[3]}$.

Developing a vehicle that can navigate autonomously in outdoor changing and dynamic agricultural environments is a challenging and difficult task and is crucial to any Intelligent Farm Vehicle developed for farming ${ }^{[4]}$.Covering a large spectrum of different technologies, as well as some of the most advanced space science and engineering ${ }^{[5]}$.

The key element of automatic guidance is navigation sensors to detect vehicle position, vehicle heading, and the current state of the vehicle (speed, wheel position, etc.), respectively. Some guidance sensors provide information for absolute positioning. Others only provide relative positioning (e.g. mechanical feelers and machine vision) between the vehicle and the guidance directrix. The usually used position sensors are mechanical feelers, machine vision and GPS sensors. And the usually used heading sensors are machine vision, GPS, with some work taking place with geomagnetic direction sensors (GDS) and inertial sensors ${ }^{[3]}$.

Patterson et al. (1985) developed a dead reckoning system for used on a towed planter with wheel encoders ${ }^{[6]}$. A Japanese company, Kubota, has developed a method of guidance by photocells in order to exploit the presence of the crop edge left by the previous passage of the vehicle (Yoshida et al., 1988) ${ }^{[7]}$. Freeland et al. (1992) experimented with a low cost electronic compass used together with wheel encoders to provide deadreckoning posture information ${ }^{[8]}$. Stanford University demonstrated high accuracy carrier phase differential GPS for the guidance of a John Deere 7800 series tractor ${ }^{[9]}$. An autonomous tractor using an optical surveying device and a terrestrial magnetism sensor for ploughing was developed (Yukumoto and Matsuo, 1995; Masuo et al., 2002) ${ }^{[10][11]}$. Carnegie-Mellon Robotics Institute developed an autonomous New Holland 2550 Speedrower for cutting forage using vision-based perception on the cut and uncut regions of the crop ${ }^{[12]}$. The University of Illinois, with international cooperation with 
Hokkaido University in Japan, has a research program for both automatic and autonomous guidance of agricultural vehicles based on two Case 8920 $2 \mathrm{WD}$ and MFD tractors ${ }^{[13]}$. Research is considering automation in terms of various posture sensors (global positioning systems, machine vision, inertial, and geomagnetic direction sensors).

Each of the approaches has its own advantages and disadvantages, which are limited by the application requirements as well as the environment where the method is going to be used.

As the fundamental element of agriculture technology, the global positioning system (GPS) signal can be used for agricultural vehicle guidance. It can provide the location, in degrees of longitude and latitude, of a receiver anywhere in the world (Langley, 1997) ${ }^{[14]}$.

Global positioning systems, although new to agriculture, have generated much of the potential growth in agricultural equipment automation by enabling the agricultural equipment industry and producers to see beyond the limitations of other navigation sensors ${ }^{[3]}$.

In this paper, give a review of previous research on automatic vehicle guidance based on GPS only or combined with local sensors.

\section{METHODS}

GPS technology has extremely important positions in the application of agriculture. By combining information collection with time, space and combining control technology and spacetime positioning, GPS has become the essential bridge between the information system and intelligent device of agricultural machinery ${ }^{[15]}$.

The Global Positioning System (GPS) is a satellite-based radio positioning navigation and real-time ranging system developed by US Department of Defense. It is a highly accurate, full-time and rapid satellite navigation positioning system built for meeting the demand of military and business application. The whole GPS system consist of 24 satellites allocated in six orbital planes. Each satellite transmits carrier wave radio signals on the L1 (1575.42MHZ) and L2 (1227.6MHZ) frequencies. The GPS satellite transmits two kinds of positioning signals: $\mathrm{P}$ code and C/A code. A GPS receiver tracks these GPS satellites and receives signals (ranges to the satellites and the satellites orbit parameters) on the basis of the work conditions of GPS visible satellites in the sky.

The main principle behind a satellite navigation system is the creation of a trilateration from any point on the earth's surface to the satellites in view. In principle, three satellites must be available to determine a three- 
dimensional position ${ }^{[17]}$. All points, which have the same distance to one satellite, form a spherical surface with the satellite in the centre. Three spherical surfaces intersect in two points. One point can be disregarded, because its position is located too far from the earth.

High-level accuracy of several centimeters is necessary for machine guidance applications. The accuracy of GPS can be improved with selective availability (SA) down to a few meters with differential GPS (DGPS). The most commonly used form of DGPS is code-differential GPS. In codedifferential GPS, the GPS receivers at the vehicle and reference station take phase measurements of the GPS pseudo-random noise (PRN) signal. The reference and vehicle measurements are then differenced to provide a relative position measurement. Code-differential systems offer 1 to $2 \mathrm{~m}$ accuracy and corrections are valid for a large radius, usually on the order of $100 \mathrm{~km}$ (Parkinson and Spilker, 1996b) ${ }^{[16]}$.

It is even possible to achieve centimeter accuracy with high-precision receivers working with carrier phase tracking techniques. A type of ultraprecise GPS positioning is known as Carrier-Phase Differential GPS, CPDGPS is based on measuring the phase of the GPS carrier signal. Because the L-band GPS carrier signal has a wavelength of approximately $19 \mathrm{~cm}$ and GPS receivers can track that carrier signal to within roughly $1 \mathrm{~cm}{ }^{[1]}$.

In the meantime, the measurement of the attitude of a vehicle with accuracy of up to $0.1^{\circ}$ is possible with multiple antenna arrangements. Such accuracy is needed for autonomous guidance (Bell, 1999) to measure and compensate vehicle movements caused by rough terrain and slopes.

Although some accurate GPS receivers are available for providing centimeter accuracy in position measurement, most low-cost GPS receivers used in agricultural vehicles normally only have meter range positioning accuracy and could not meet the navigation accuracy requirement for many precision agricultural operations and the GPS performs poorly in highly dynamic environments due to the shadowing of satellites and high rates of changes in doopler. To solve these problems, the integration of two positioning methods, relative positioning using a dead-reckoning system(odometry, inertial sensors and other sensors which measure the rotation of vehicle or implement wheels, combination of travelled distance with the measured heading or steering angle is used to estimate position from a known starting point ${ }^{[3]}$ ), and absolute positioning using a global positioning system (GPS), can be used to provide improved navigation accuracy for agricultural vehicles.

Although receiving less attention, odometery is probably the most widely used navigation sensor. The rotation of one or more ground wheels is used to measure vehicle motion. The appeal of odometry is that is simple and low cost. The disadvantage is susceptibility to errors from a variety of sources, 
including wheel slippage and variation in the effective wheel rolling radius and the track width of the wheels. These errors are cumulative, giving a rapid loss in positional accuracy.

Inertial sensors, i.e. accelerometers and gyroscopes, have been used in a number of vehicle applications as an alternative to odometers for dead reckoning. Inertial systems have the advantage that they can be produced in an encapsulated and robust package, can supply not only continuous vehicle position and velocity information but also three axes attitude ${ }^{[17]}$.

Unfortunately, both the zero point and the output scale of these types of sensor are prone to thermal drift (Barshan and Durrant-Whyte, 1993) ${ }^{[20]}$ to a varying degree depending upon the type of the sensor (and inevitably linked to $\operatorname{cost}$ ). Moreover, accelerometers are sensitive not only to accelerations of the vehicle, but also to the gravitational acceleration; therefore estimation of attitude for the full three rotational degrees of freedom is necessitated.

The use of a geomagnetic compass is also possible, a three axis fluxgate magnetometer allows an implementation with no moving parts.

In summary, dead reckoning, which determines machinery position by calculating the travel distance in the direction of machinery moving accumulatively, can provide a robust and accurate position measurement over a short period of time, improved navigation accuracy especially when GPS is degraded or interrupted because of jamming or interference ${ }^{[18]}$. But will always result in substantially accumulated positioning errors as the measurement time increases due to sensor drifting and machinery slippage. As comparison, in the GPS measurements, there is no accumulated positioning error because each measurement is independent to the previous measurement. The integration of GPS and inertial positioning can provide a complementary correction to improve both the accuracy and robustness in mobile positioning and navigation for agricultural machinery applications ${ }^{[19]}$.

\section{APPLICATIONS}

Several studies have explored the use of high accuracy GPS or the integration of GPS and dead reckoning for vehicle guidance.

Researchers at the University of Illinois (Stombaugh et al., 1998) ${ }^{[2]}$ utilized a $5 \mathrm{~Hz}$ real-time kinematic (RTK) GPS for guidance of a 2 WD Case 7720 tractor. In order to eliminate lag in the system responses the GPS was mounted in front of the front wheels on a mast extending to a height above the cab. Vehicle response in tracking a 3-m step change in position showed that the lateral position error at $4.5 \mathrm{~m} / \mathrm{s}$ was within $16 \mathrm{~cm}$ ( $95 \%$ confidence). 
Benson et al. (1998) used GDS and GPS together for vehicle guidance based on dead reckoning as a simple path planner. The system was tested at slow speeds $(1.12 \mathrm{~m} / \mathrm{s})$ and had an average error of less than $1 \mathrm{~cm}$, which compared favorably to GPS-based guidance. When implementing a 3-m step change in responses the sensor fusion system had a maximum overshoot of $12 \%$. Under GPS-under mode the system experienced a $50 \%$ overshoot ${ }^{[21]}$.

A low-cost integrated positioning system, consisting of a Garmin GPS and a set of solidstate inertial sensors, was developed for autonomous agricultural vehicle applications. Based on a position-velocity-attitude (PVA) model of the vehicle, a PVA model-based Kalman filter was designed to integrate data from all sensors to provide accurate and robust vehicle positioning data. Figure 1 shows the block diagram of the PVA model-based fusion algorithm for the integrated position sensing system.

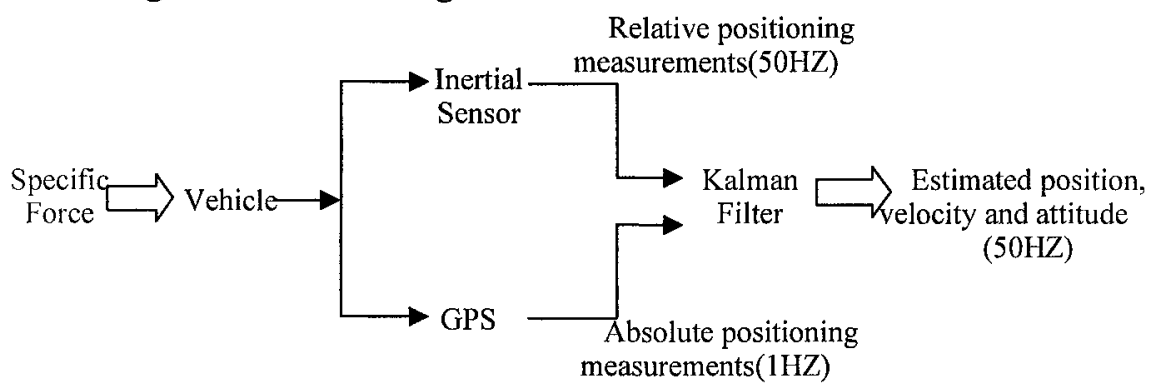

Figure 1. PVA model-based GPS/Inertial sensor fusion

This integrated positioning system was evaluated in different test sites. Test results showed that the maximum positioning error was $0.50 \mathrm{~m}$ while the vehicle was traveling on a known path (determined based on static RTKDGPS measurements) on a paved road nearby buildings and partially under trees. When the vehicle was traveling on open field, the maximum error was $0.30 \mathrm{~m}$. The accuracy of this low-cost integrated positioning system can meet most tasks in precision farming applications ${ }^{[22]}$.

\section{CONCLUSIONS}

Current research has proven the basic concept of vehicle automation for agriculture and an overview has been presented of the subject of agricultural vehicles navigation using GPS signals. From successful experiments of past and on-going projects, it can be concluded that GPS may be successfully used as absolute positioning sensor on agricultural vehicles. However, the accuracy will in general not be high. For highly accurate and robust real time attitude determination, GPS equipment needs additional low-cost relative positioning sensors to assist in increasing the data rate and robustness. The 
future of automation and robotics in production agriculture is entering an exciting era. The potential for automatic and autonomous agricultural machines continues to grow at a rapid pace. The use of differential techniques, important for applications of machine guidance, as well as for attitude measurement will be possible, the on-going activities in GPS will improve the use of satellite navigation in Agriculture, the paths of university research, industrial development and government policies are on a course that will lead to increased opportunities for automation in agriculture ${ }^{[23][24]}$.

\section{ACKNOWLEDGEMENTS}

This study was supported by National High Technology Research and Development Program of China (863 Program): Research and Demonstration for Digital Agriculture (2003AA209040).

\section{REFERENCES}

[1] Thomas Bell, 1999. Automatic tractor guidance using carrier-phase differential GPS. Computers and Electronics in Agriculture : Special Issue Navigating Agricultural Field Machinery. 25 (2000) 53-66

[2] T. S. Stombaugh, E. R. Benson, J. W. Hummel,1998. AUTOMATIC GUIDANCE OF AGRICULTURAL VEHICLES AT HIGH FIELD SPEEDS. ASAE Paper UIUC-ENG 98-7002. St. Joseph, MI

[3] . John F. Reid a, Qin Zhang a, Noboru Noguchi b,Monte Dickson. Agricultural automatic guidance research in North America. Computers and Electronics in Agriculture 25 (2000) 155-167

[4] . HANI HAGRAS, MARTIN COLLEY AND VICTOR CALLAGHAN, Online Learning and Adaptation of Autonomous Mobile Robots for Sustainable Agriculture. Autonomous Robots 13, 37-52, 2002

[5] agarajan Ramalingam, Timothy S. Stombaugh, and Jonathan Mirgeaux Ramalingam, N., T. S. Stombaugh, J. Mirgeaux. 2000. DGPS-Based Automatic Vehicle Guidance. ASAE Paper No. 001068. Annual International Meeting, Midwest Express Center, Milwaukee, Wisconsin, July 9-12.

[6] Patterson, R.J., Fehr B.W., Sheets. L.P., 1985. Electronic guidance system for a planter. ASAE Paper No. 85-1587. St. Joseph, MI:ASAE.

[7] Yoshisada Nagasaka, Naonobu Umeda, Yutaka Kanetai, Ken Taniwaki, Yasuhiro Sasaki, Autonomous guidance for rice transplanting using global positioning and gyroscopes. Computers and Electronics in Agriculture 43 (2004) 223-234 
[8] Freeland, S.R., Wilkerson B.J., Hart. E.W., 1992. Instrumentation for measurement of tractor yaw. ASAE Paper No. 92-3552. ASAE, St. Joseph, MI.

[9] O'Connor, Michael, Thomas Bell, Gabe Elkaim, and Bradford Parkinson. 1996. Automatic steering of farm vehicles using GPS. 3rd International Conference on Precision Agriculture, Minneapolis, MN, June 23-26.9.

[10] Yukumoto, O., Matsuo, Y., 1995. Research on autonomous land vehicle for agriculture. in: Proceedings of the International Symposium on Automation and Robotics in Bioproduction and Processing, vol. 1, JSAM, Kobe, pp. 41-48.

[11] .Masuo, Y., Yamamoto, S., Yukumoto, O., 2002. Development of tilling robot and operation software.in: Proceedings of the Automation Technology for Off-road Equipment, ASAE, Chicago, IL, pp. 184-189.

[12] Ollis, M. and A. Stentz. 1997. Vision-based perception for an automated harvester. IROS'97.

[13] Will, J., T. Stombaugh, E. Benson, N. Noguchi, and J.F. Reid. Development of a flexible platform for agricultural guidance research. ASAE paper 983202. St. Joseph, MI.

[14] Langley, R.B. 1997. GLONASS: review and update. GPS World 8(7):46-55.

[15] Zhang XiaoChao, Wang Yiming, Ma Zhiyuan, Li liang. The Study of GPS Based Irrigation System for Precision Agriculture Technology. Transaction of the CIGR 2004 Paper No.30-109

[16] .Parkinson, B.W., Spilker, J.J. (Eds.), 1996a. Global Positioning System: Theory and Applications, American Institute of Aeronautics and Astronautics, Vol. I. 1996b. Global Positioning System: Theory and Applications,American Institute of Aeronautics and Astronautics, Vol. II.

[17] Lin Ninmin, Fang Jiancheng, Gan Guojian. A NEW COMPOSED CORRECTING KALMAN FILTERING METHOD FOR GPSISINS IN TEGRATED NAVIGATION SYSTEM

[18] Ma ruiping, Zhang minglian. A NEW ADAPTVIE KALMAN FILTER FOR GPS/INS INTEGRATED SYSTEM.

[19] T. Hague, J.A. Marchant, N.D. Tillett. Ground based sensing systems for autonomous agricultural vehicles. Computers and Electronics in Agriculture 25 (2000) 11-28

[20] Barshan, B., Durrant-Whyte, H.F., 1993. An inertial navigation system for a mobile robot. In:IEEE:RSJ International Conference Intelligent Robot Systems, pp. 2243-2248.

[21] Benson, E., Stombaugh T., Noguchi N., Will J., Reid. J.F., 1998. An evaluation of a geomagnetic direction sensor for vehicle guidance in precision agriculture applications. ASAE paper UILU 98-7011. St. Joseph, MI.

[22] L.S. Guo, Q. Zhang,L. Feng. A Low-Cost Integrated Positioning System of GPS and Inertial Sensors for Autonomous Agricultural Vehicles. ASAE Paper Number: 033112, St. Joseph, MI

[23] Dr. John F. Reid .A Status Report on Autonomous Guidance of Agricultural Vehicles in the US: New Frontiers in the 21st Century. UILU-ENG-987026

[24]Q.P.Chu,P.Th.L.M.vanWoerkon. GPS for Low-Cost Attitude Determination. Acta Astronautica Vol.41,Nos 4-10,pp.421-433,1997 